\title{
Modulation of Yeast-Derived Volatile Aromas by Oleic Acid and Sterols
}

\author{
S. Fairbairn ${ }^{1 *}$, A. C. Silva Ferreira ${ }^{1,2}$, F. F. Bauer ${ }^{1}$ \\ (1) Institute for Wine Biotechnology, Department of Viticulture and Oenology, University of Stellenbosch, Stellenbosch South \\ Africa \\ (2) Escola Superior de Biotecnologia, Universidade Católica Portuguesa, Rua Dr. António Bernardino de Almeida, $4200-072$ \\ Porto, Portugal
}

Submitted for publication: October 2018

Accepted for publication: March 2019

Keywords: volatile aroma, oleic acid, ergosterol, yeast growth, $\beta$-sitosterol

\begin{abstract}
Unsaturated fatty acids and sterols are essential constituents of the yeast plasma membrane. Recently, their contribution to modulating the production of yeast-derived volatile compounds has received significant attention. The objective of this study was to determine how sterol and lipid supplementation, including ergosterol, plant sterols or oleic acid, differentially influenced yeast growth as well as the production of fermentative aromas when added individually or in combinations. Oleic acid significantly altered the volatile profiles produced and lowered yeast growth. Generally, phytosterol ( $\beta$-sitosterol) and ergosterol supplementation resulted in similar responses regarding the production of aromas, however, they differed in the magnitude of the response in the case of medium chain fatty acids and acetate esters synthesis. The combinations of sterols with oleic acid resulted in a response more closely associated with the oleic acid control treatment, showing lower levels of acetate ester production.
\end{abstract}

\section{INTRODUCTION}

Plasma membrane composition plays an essential role in cell growth and survival (Daum et al., 1998). Its continuous adaptation to changing environmental conditions is essential for cellular growth and stress resistance, including in biotechnologically relevant processes such as anaerobic batch fermentation. Changes in plasma membrane composition maintain membrane integrity and function in response to fermentation stresses such as high sugar concentrations and increasing levels of ethanol (Daum et al., 1998).

One of the mechanisms of adaptation is the modulation of membrane fluidity by adjusting the concentrations of sterols and unsaturated fatty acids found in phospholipids (You et al., 2003; Mannazzu et al., 2008). Changes in fluidity may counter the deleterious effect of compounds such as ethanol which increase membrane permeability and negatively impact the transport or retention of protons and of essential nutrients including amino acids (Piper, 1995; Ma \& Liu, 2010). There are however conflicting reports regarding the importance of ergosterol content on ethanol tolerance, as high ergosterol content is not always correlated with improved tolerance (You et al., 2003; Aguilera et al., 2006; Mannazzu et al., 2008; Redón et al., 2011). In contrast, ethanol tolerant yeast cells generally contain higher levels of unsaturated fatty acids (You et al., 2003).
Oxygen is essential for both ergosterol formation and the desaturation of fatty acids (Snoek \& Steensma, 2007). Saccharomyces cerevisiae only produces monounsaturated fatty acids via the action of a delta- 9 fatty acid desaturase, and the most prevalent fatty acids in yeast cells are palmitic acid (C16:0), palmitoleic acid (C16:1), stearic acid (C18:0), and oleic acid (C18:1) (Daum et al., 1998). A mutation in the OLE1 gene encoding this desaturase results in mutants displaying limited growth in the absence of exogenous unsaturated fatty acids due to their re-distribution across daughter cells (Stukey et al., 1989). Interestingly, this growth limiting effect is mitigated by supplementation with exogenous unsaturated fatty acids. During alcoholic fermentation, oxygen is rapidly depleted, and the cell can no longer manufacture these protective compounds. However, cells continue to assimilate sterols and fatty acids from the environment, and a significant number of studies have evaluated the impact of ergosterol, the only sterol produced by yeast, phytosterols (b-sitosterol, campesterol and stigmasterol (Delfini et al., 1993; Luparia et al., 2004; Rollero et al., 2014; Ochando et al., 2017) and unsaturated fatty acids (Thurston et al., 1981; Duan et al., 2015).

Relatively few quantitative surveys of free sterol and unsaturated fatty acid levels in grape must are available (Arita et al., 2017). Data show that the levels of both types of

*Corresponding author: E-mail address: fairbairn@sun.ac.za
Acknowledgements: The authors thank the National Research Foundation (NRF) of South Africa and Winetech, the research funding body of the South African
wine industry, for funding this project. The authors would also like to thank Dr Hélene Nieuwoudt for establishing the synthetic grape must prediction models 
compounds vary significantly and are strongly affected by the winemaking practices. In particular, levels may decrease dramatically after clarification (Delfini et al., 1993; Cocito \& Delfini, 1997; Varela et al., 1999), as clarification removes the lipid-containing grape solids (Casalta et al., 2016) Nonetheless, after clarification, phytosterols have been reported at levels ranging between $6 \mathrm{mg} / \mathrm{L}$ and $50 \mathrm{mg} / \mathrm{L}$ (Delfini et al., 1993; Cocito \& Delfini, 1997) and free fatty acids ranging from 0 to $28.45 \mathrm{mg} / \mathrm{L}$ (Varela et al., 1999; Tumanov et al., 2015).

Previous studies evaluating the impact of lipids have reported that lipid supplementation, both as a direct addition (Lafon-Lafourcade et al., 1979; Taylor et al., 1979; Luparia et al., 2004; Ochando et al., 2017) or in the form of grape solids (Casalta et al., 2013), may improve yeast growth and consequently fermentation kinetics, and directly triggers changes in the plasma membrane composition (Thurston et al., 1981; Mauricio et al., 1998; Luparia et al., 2004). In addition, impacts on the production of volatile compounds have been reported (Thurston et al., 1981; Mauricio et al., 1997; Rollero et al., 2014; Duan et al., 2015; Rollero et al., 2016). These data show that unsaturated fatty acid supplementation can result in an increase in higher alcohol and a decrease in acetate ester production. This reduction can be alleviated by the supplementation of higher concentrations of more complex unsaturated fatty acid mixtures (oleic $>$ $50 \mathrm{mg} / \mathrm{L}$, linoleic acid $>150 \mathrm{mg} / \mathrm{L}$ and a-linolenic acid $>$ 40mg/L) (Duan et al., 2015). Similarly, supplementation with sterols increased the production of volatile aroma compounds from most volatile aroma families (Mauricio et al., 1997; Varela et al., 2012). However, the complex interactions between these growth-supporting or stress-resistance inducing compounds, fermentation kinetics and secondary metabolism remains not fully understood. Understanding these connections better is of significant relevance to wine production, as lipid additions may only be made in the form of grape solids (Casalta et al., 2013, 2016) or various products such as inactive dried yeast which are specifically formulated to significantly impact on sterol and fatty acid composition (Sablayrolles, 2009). As the cell is able to assimilate exogenous sterols and unsaturated fatty acids in addition to synthesizing ergosterol during winemaking, this study explores whether combinations of yeast (ergosterol) and plant $(\beta$-sitosterol) derived sterols together with oleic acid differently affect yeast growth and volatile aroma production. The aroma profiles were evaluated using targeted chemical analyses. Fermentations were conducted in synthetic grape must supplemented with ergosterol, phytosterol (b-sitosterol), and oleic acid independently as well as in combination with each other.

\section{MATERIALS AND METHODS}

\section{Fermentation media, conditions, and treatments}

Saccharomyces cerevisiae yeast strain, VIN13 (Anchor Yeast, Cape Town, South Africa), was rehydrated (20 g/hL) according to the supplier's instructions. Briefly, VIN13 was rehydrated for $20 \mathrm{~min}$ at $37^{\circ} \mathrm{C}$ in warm water and subsequently cooled to within $10^{\circ} \mathrm{C}$ of the media's temperature before inoculation.

Fermentations $(200 \mathrm{~mL})$ were conducted in triplicate us- ing synthetic grape must (100 g/L glucose and $100 \mathrm{~g} / \mathrm{L}$ fructose), as previously described (Henschke \& Jiranek, 1993). The nitrogen sources (amino acids and ammonium) were added at a concentration of $200 \mathrm{mg} / \mathrm{L}$ of fermentable nitrogen (Bely et al., 1990). Fermentations were treated from a ten times stock with anaerobic factors $(20 \mathrm{mg} / \mathrm{L})$ : phytosterol ( $\beta$-sitosterol, campesterol, stigmasterol), ergosterol, and oleic acid, and contained $10 \mathrm{mg} / \mathrm{L}$ of each constituent when used in combinations. In subsequent sections, this phytosterol treatment is referred to as b-sitosterol (PHY) (85451, Sigma Aldrich), as it is the primary constituent. The sterols were provided at, at least, twice the proposed minimum concentration required for maximal fermentation and population development at $10 \mathrm{mg} / \mathrm{L}$, (Luparia et al., 2004), which falls within levels reported in natural grape juice (Delfini et al., 1993; Cocito \& Delfini, 1997). Oleic acid was provided within average levels reported in grape juice (Tumanov et al., 2015) and also at a dosage great enough to detect treatment effects. Anaerobic factors were dissolved in ethanol and the untreated control received an addition of the same volume of ethanol only $(2 \mathrm{~mL})$.

Self-generated anaerobiosis was supported by replacing the oxygen in the headspace with sterile nitrogen, and fermentations conducted in modified Erlenmeyer flasks fitted with a sample port and sealed with a septum. The flasks were sealed with rubber stoppers and a $\mathrm{CO}_{2}$ outlet was provided. Static fermentation took place at $20^{\circ} \mathrm{C}$, and fermentation progress was monitored twice a day for the first week and daily thereafter by weight loss, and growth by OD (600 nm) and plate counts $(\mathrm{CFU} / \mathrm{mL})$ in addition to residual sugar levels (ATR FT-MIR). Fermentations were considered complete with the residual sugars were below $4 \mathrm{~g} / \mathrm{L}$.

\section{ATR FT-MIR spectra collection}

Spectra, from triplicate fermentation samples, were collected on an Alpha-P FT-MIR ATR instrument fitted with a heatable diamond crystal sample plate (Bruker Optics, Ettlingen, Germany). The absorbance spectrum of each sample was obtained at $40{ }^{\circ} \mathrm{C}$ by averaging 128 repeated scans at a resolution of $4 \mathrm{~cm}^{-1}$ and in the range 4000 to $600 \mathrm{~cm}^{-1}$, with a scanner velocity of $7.5 \mathrm{KHz}$. Prior to scanning, a reference background measurement was taken against water. The instrument was operated using OPUS software version 7 (Bruker Optics, Ettlingen, Germany). In-house prediction models established for synthetic grape must were used to quantify the glucose, fructose, glycerol and ethanol levels as a means to monitor alcoholic fermentation.

\section{Gas chromatography-flame ionization detector (GC- FID)}

At the end of alcoholic fermentation, samples underwent a liquid-liquid extraction as previously described for analysis by gas chromatography (Louw et al., 2009). A 5-mL sample of the fermented media, $100 \mu \mathrm{l}$ of internal standard (4-methyl-2-pentanol, $0.5 \mathrm{mg} / \mathrm{L}$ ) and $1-\mathrm{mL}$ of solvent (diethyl ether) were combined and this mixture was placed in an ultrasonic bath for 5 minutes to facilitate extraction. The mixture was centrifuged for $3 \mathrm{~min}$ at $4000 \mathrm{rpm}$ after which $\mathrm{Na} \mathrm{SO}_{4}$ was added to remove any water from the non-polar layer and the sample was again centrifuged for another 3 
min at 4000 rpm. A Hewlett Packard 6890 Plus GC-FID instrument (Agilent, Little Falls, Wilmington, USA) with a split/splitless injector was used for major volatiles quantification. The split flow rate was set at $49.4 \mathrm{ml} / \mathrm{min}$ and the split ratio was set to $15: 1$ at a temperature of $200^{\circ} \mathrm{C}$. The separation of compounds was done using a $\mathrm{J}$ and B DBFFAP capillary GC column (Agilent, Little Falls, Wilmington, USA) with the dimensions of $60 \mathrm{~m} \times 0.32 \mathrm{~mm}$ and a $0.5 \mu \mathrm{l}$ coating film thickness with the flow rate of the hydrogen carrier gas set at $3.3 \mathrm{ml} / \mathrm{min}$. An initial oven temperature of $33^{\circ} \mathrm{C}$ was held for $8 \mathrm{~min}$; the temperature was then increased by $21^{\circ} \mathrm{C} /$ min to $130^{\circ} \mathrm{C}$ and then held for $7 \mathrm{~min}$; increased by $12^{\circ} \mathrm{C} /$ min to $170^{\circ} \mathrm{C}$ and held for $5 \mathrm{~min}$; increased by $21^{\circ} \mathrm{C} / \mathrm{min}$ to $240^{\circ} \mathrm{C}$ and held for $2.5 \mathrm{~min}$. Once the FID oven temperature reached the temperature of $240^{\circ} \mathrm{C}$, three microliters of the extracted sample was injected into the gas chromatograph. A post-run step at the end of each sample was carried out at $240^{\circ} \mathrm{C}$ for $5 \mathrm{~min}$. The column was cleaned with an injection of hexane after every 10 samples. Authentic reference standards (Merck, Cape Town) were used to calibrate for each of the compounds using the internal standard compound 4-methyl-2-pentanol $(10 \mathrm{mg} / \mathrm{L})$. Manual data collection and peak integration were done using the HP ChemStation software (Rev. B01.03 [204]).

\section{Data analyses}

Analysis of variance (ANOVA) was performed using XLSTAT 2017 (Addinsoft, Paris, France), using Fisher's LSD for the post-hoc testing $(p<0.05)$. ANOVA was performed on the primary metabolite concentrations (glucose, fructose, glycerol and ethanol), of 3 biological replicates, measured throughout fermentation, as well as the GC-FID volatile chemical data determined at the end of alcoholic fermentation. Those volatile compounds identified as not being significantly $(\mathrm{p}<0.05)$ influenced by the lipid treatments were omitted from the subsequent principal component analyses (PCA). This PCA was also performed with the XLSTAT 2017 (Addinsoft, Paris, France). The GC-FID volatile chemical data was also normalised by the average maximum optical density measured before undergoing PCA analyses.

The heatmaps were used to compare the overall changes in the production of volatile aromas in response to lipid supplementation both before and after normalization by yeast growth (OD). Cluster analysis was also performed on the heatmaps, using the Ward method to determine the linkage between treatments and Pearson's correlation coefficient was used as the distance measure. For the heatmap, the volatile acidity (ethyl acetate and acetic acid), total higher alcohols, acetate esters, short chain fatty acids (SFA), SFA ethyl esters, medium chain fatty acids (MFA) and MFA ethyl esters concentrations were autoscaled and the heatmap was constructed using the package Complex Heatmap in R studio

\section{RESULTS AND DISCUSSION}

\section{Fermentation and growth kinetics}

Figure 1 illustrates the sugar consumption and biomass production for the different lipid treatments. Supplementation with ergosterol (Erg) and $\beta$-sitosterol (Phy), independently $(20 \mathrm{mg} / \mathrm{L})$ or in combination with each other (Erg+Phy) or oleic acid (Erg+Ole and Phy+Ole) $(10 \mathrm{mg} / \mathrm{L}$ each $)$ signifi- cantly improved sugar consumption and yeast growth relative to the untreated control. Interestingly, during the first 2 days, fermentations supplemented with both the sterol and oleic acid (Erg+Ole and Phy+Ole) displayed an improvement in sugar consumption compared to the sterol alone, however, this competitive advantage was subsequently lost by the fermentation mid-point. Nonetheless, this suggests that the oleic acid addition improved the initial rates of sugar consumption even though sterol levels alone were sufficient to support growth. Luparia and colleagues have shown that as little as $5 \mathrm{mg} / \mathrm{L}$ of phytosterol or ergosterol were able to result in similar maximal fermentation rates and biomass sizes in the absence of oxygen (Luparia et al., 2004). However, they also found that the fermentations treated only with phytosterol displayed a comparative loss in cell viability towards the end of alcoholic fermentation. As shown in figure 1, no such loss in viability is apparent which is possibly due to the lower sugar content of the synthetic grape must used here (200 compared to $240 \mathrm{~g} / \mathrm{L}$ ) as well as their more stringent anaerobic conditions (Luparia et al., 2004). Nonetheless, all sterol treatments completed fermentation in 11 days whereas the untreated control and the oleic acid treatment required additional time.

Generally, data trends suggest that oleic acid supplementation lowered the initial yeast growth, however, the oleic acid only treatments resulted in a population size similar to that of the untreated control during the latter stages of fermentation (Fig. 1). Moreover, at the end of alcoholic fermentation, the data suggests that oleic acid supplementation improved yeast viability. Interestingly, as seen in combination with sterols, the addition of oleic acid and phytosterol significantly improved the rate of sugar consumption during the first third of alcoholic fermentation. This affirms that the addition of unsaturated fatty acids provides a competitive advantage to cells (Taylor et al., 1979; Varela et al., 2012; Duan et al., 2015), possibly allowing them to adapt to the environment more rapidly resulting in a more effective initial consumption of sugar.

Limited growth in the presence of oleic acid was apparent at even low ethanol levels $\left(2.5\right.$ to $3 \%$ ( $\left.\mathrm{vol}_{\mathrm{vol}}\right)$ ethanol), suggesting that at this concentration unsaturated fatty acids alone cannot compensate for a sterol deficiency. Indeed, the absence of oxygen means that the limited amount of sterols and unsaturated fatty acids were influential in determining the number of cell divisions possible (Henry, 1982; Rosenfeld et al., 2003; Zara et al., 2008). Nonetheless, at the end of alcoholic fermentation, all treatments resulted in similar glycerol $(5.26 \pm 0.11 \mathrm{~g} / \mathrm{L})$ and ethanol $(12.78 \pm 0.12 \%)$ levels.

\section{Volatile aroma profiles}

At the end of alcoholic fermentation, the fermented media underwent chemical analysis by GC FID (Table 1). Principal component analysis (PCA) was performed on the chemical data to evaluate whether the lipid treatments had a reproducible impact on the production of aromatic compounds (Fig. 2A). The PCA suggests that relative to the untreated control, both the oleic acid and sterol treatments may be influential in determining the volatile aromas produced. The first PC differentiates between treatments $(47.8 \%)$ based on 
(A)

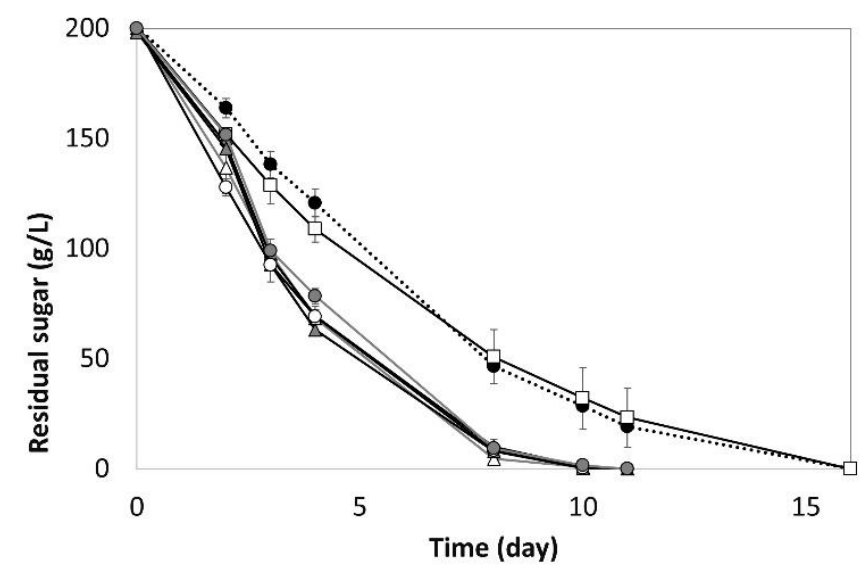

(B)
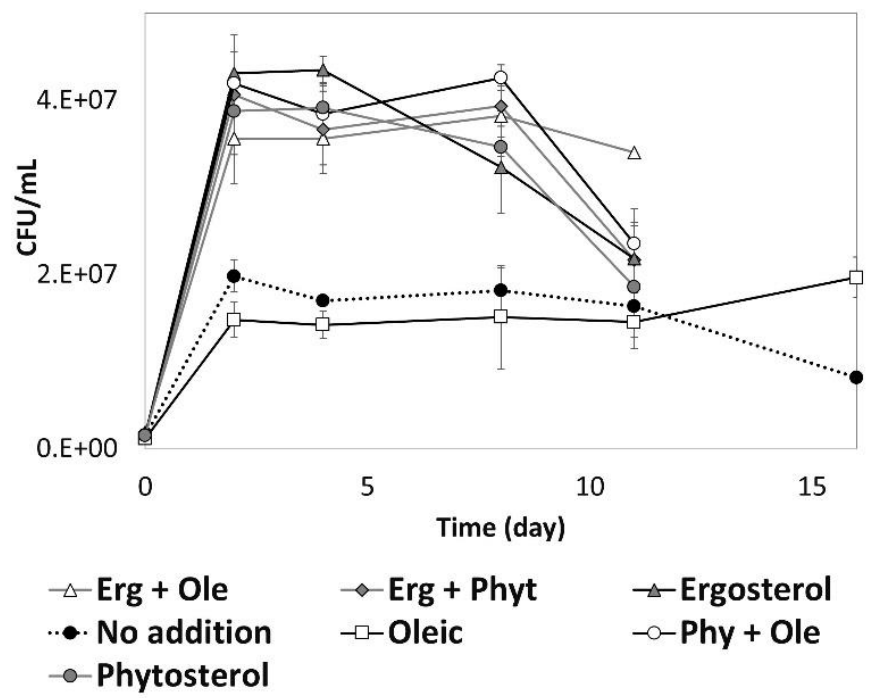

FIGURE 1

The impact of anaerobic factors on residual sugar concentrations (g/L) (A), as well as CFU/mL (B) of VIN13. The anaerobic factor treatments were as follows: ergosterol (Erg), b-sitosterol (Phy), and oleic acid (Ole) used independently in addition to their use in combinations with each other, relative to an untreated control.

(A)

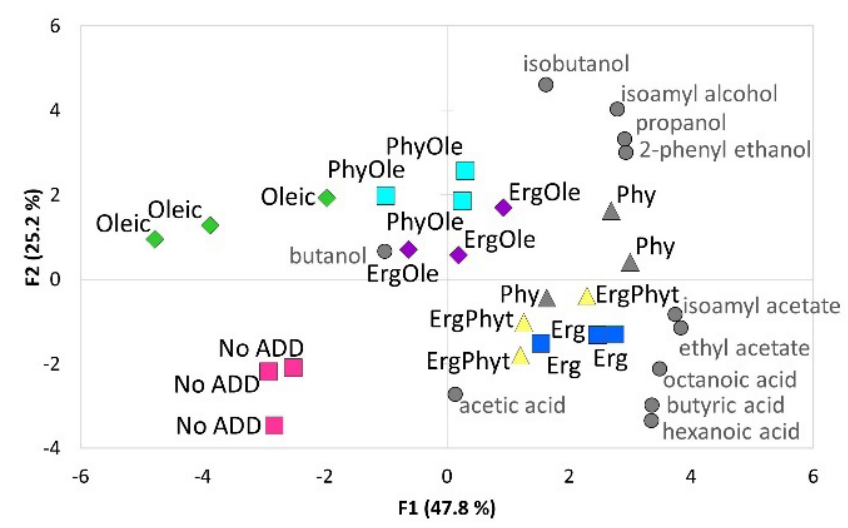

(B)

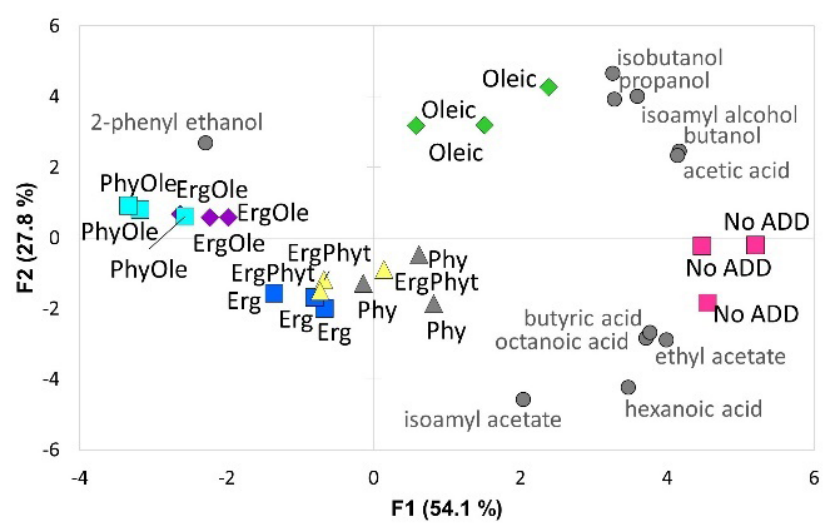

FIGURE 2

Principal component analyses of the volatile data (GC-FID) in response to various anaerobic factor treatments before (A) and after normalization with the average maximum optical density (B). The treatments include ergosterol (Erg), b-sitosterol (Phy), and oleic acid (Ole) added independently, in addition to their use in combinations with each other, as well as a no addition

(No ADD) control.

the absence or presence of sterols, which is at least in part due to the relative improvement in growth observed in the sterol supplemented media, as the factors which alter yeast growth also influence the production of volatile compounds (Dufour et al., 2003). The second principal component (25.2\%) separates those fermentations treated with oleic acid from the remaining treatments, suggesting that oleic acid alters the metabolic flux in a manner independent of the oleic acid treatment composition. Overall, the absence of supplementation or supplementation with only oleic acid led to low levels of volatile compounds, with some subtle differences between the two treatments (Fig. 2A). The fermentations treated with oleic acid had comparatively lower levels of acetate esters, volatile fatty acids, and their ethyl esters (Table 1). This trend was also observed when oleic acid was paired with sterols, as these treatments displayed a similar reduction in acetate esters, volatile fatty acids, and their ethyl esters.

In a winemaking context, the concentrations of aromatic compounds at the end of alcoholic fermentation is of prima- 


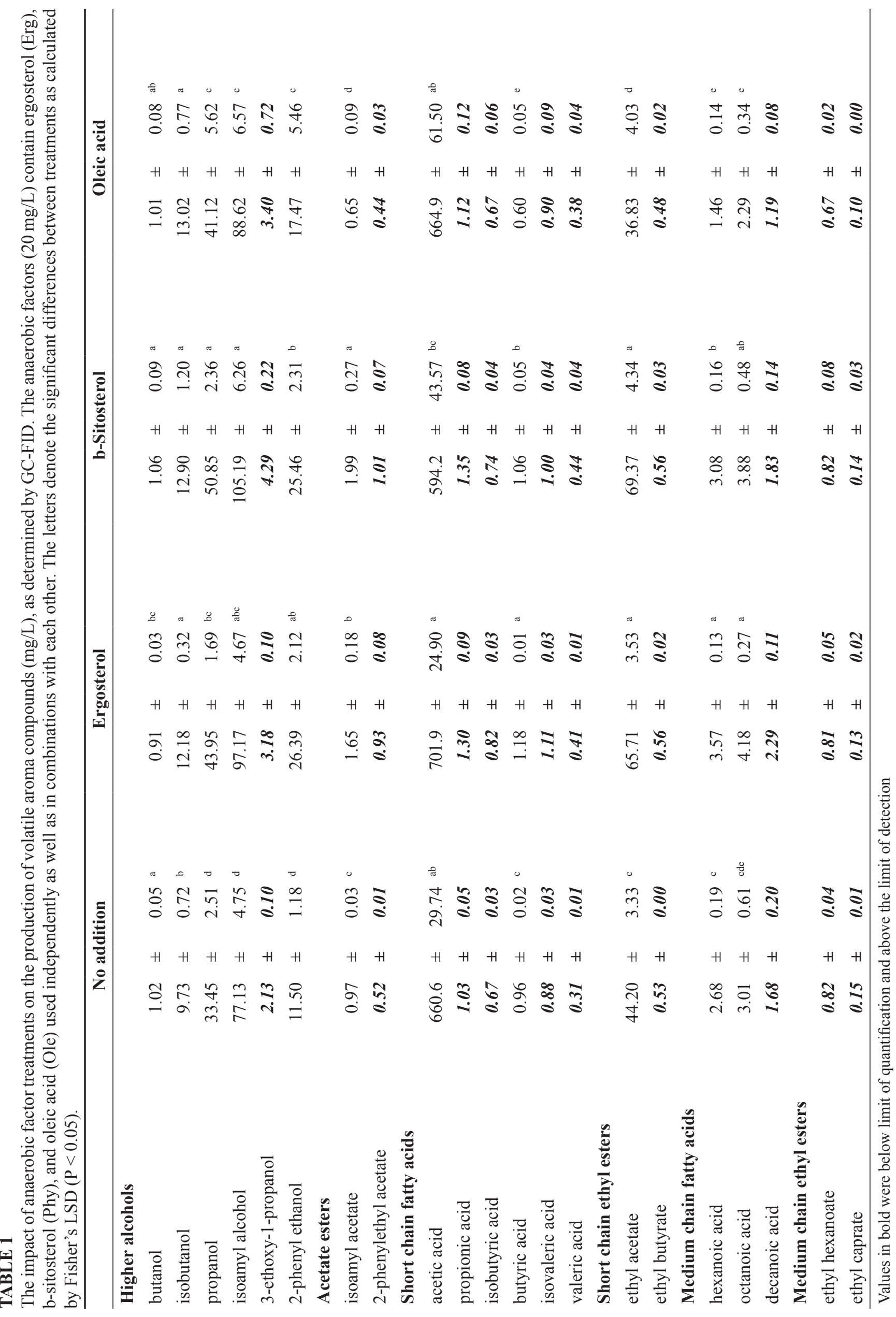




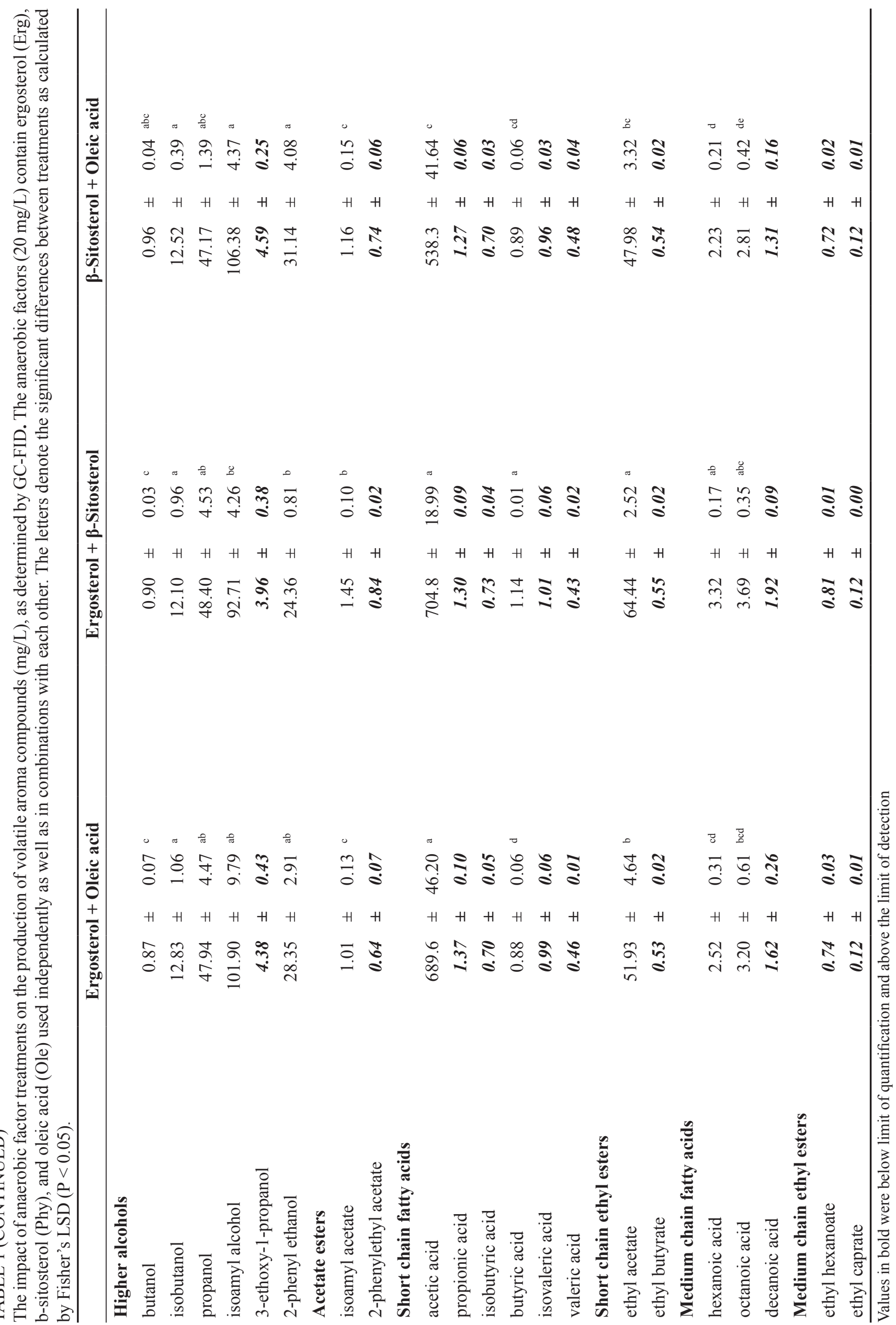


ry interest. However, the impact of the treatments was also normalized for biomass as determined by O.D. ${ }_{600}$ measurements, to determine the impact of these treatments on the cell. In this case, and similar to figure 2 , the first PC differentiates between treatments $(54.1 \%)$ based on the absence or presence of sterols (Fig. 2B), whereas the second principal component $(27.8 \%$ ) separates the fermentations treated with oleic acid from the remaining treatments. However, the relative positioning of treatments in Figure 2B have swapped in $\mathrm{PC} 1$, with the untreated control showing a greater association with volatile fatty acids and their ethyl esters, whereas, the oleic acid showed an association with higher alcohols. This suggests that the untreated control activated fatty acid production to improve stress tolerance but this was limited by a shortage of oxygen.

\section{Higher alcohols and acetate esters}

In subsequent sections, the volatile aroma data collected at the end of alcoholic fermentation as well as after normalisation with yeast biomass will be discussed. When considering the concentrations of the volatile compounds determined at the end of alcoholic fermentation, the data suggests that, oleic acid had a profound impact on the production of aromatic compounds. When added on its own, it resulted in a $23 \%$ increase in propanol and a $52 \%$ increase in 2-phenyl ethanol compared to the untreated fermentations (Table 1). Generally, fermentations supplemented with sterols, depending on the composition, increased the production of propanol between 31 to $52 \%$, isoamyl alcohol between 20 and $38 \%$, and 2-phenyl ethanol between 111 to $170 \%$ (Fig. 3A, Table 1). This positive correlation between higher alcohol production and sterol content has been observed before for both ergosterol and phytosterol (Varela et al., 2012; Rollero et al., 2014).

However, when comparing yields after endpoint data has been normalised by biomass, data show that yeast cells treated with oleic acid as well as the no addition control produced comparatively more higher alcohols than the sterol treatments (Fig. 4B). As in the case of isoamyl alcohol, which showed a comparative decrease ranging between 18 and 30\% depending on sterol treatment (Fig. 3C, 4B). This suggests that the positive impact of sterols is primarily linked to biomass production, and not because of metabolic regulatory impacts. A possible explanation for the higher yield in the control and the presence of oleic acid is that the yeast also produced comparatively more acetic acid per cell (Fig. 4B) and that higher alcohol production was favoured to restore the redox balance.

The fact that $\beta$-sitosterol and ergosterol similarly impacted these metabolic pathways suggests that they are incorporated into cell membranes similarly (Luparia et al., 2004). However, phytosterol has been shown to disrupt cell membranes when it is the dominant sterol in high sugar musts resulting in poor viability during the latter stages of alcoholic fermentation. Nonetheless, it is possible that the presence of these sterols aid in amino acid retention following exposure to ethanol (Piper, 1995).

Amino acids or alpha-keto acids are metabolized via the Ehrlich pathway, resulting in the formation of either higher alcohols or fusel acids, depending on the redox state of the cell (Hazelwood et al., 2008). Subsequently, the higher alcohol may react with acetyl-CoA to produce an acetate ester. The availability of these substrates, acetyl-CoA, and an alcohol group, in addition to the activity of alcohol acetyl transferase (ATF1 and $A T F 2$ ) enzymes, influence acetate ester formation (Yoshioka \& Hashimoto, 1981; Saerens et al., 2010). Furthermore, the expression of ATF1 is repressed by the presence of unsaturated fatty acids and oxygen (Fujii et al., 1997). The end of alcoholic fermentation data shown here (Table 1) clearly indicates that the addition of oleic acid, when used independently or in combination with sterols, resulted in a relative decrease in the production of ethyl acetate and isoamyl acetate. This decrease in acetate ester formation may be due to the repression of ATF1. As the formation of acetate esters is hindered by the presence of unsaturated fatty acids, an accumulation of higher alcohols would be expected. It is important to keep in mind that a significant change in acetate ester levels, such as isoamyl acetate present at $0.65 \mathrm{mg} / \mathrm{L}$ in the oleic acid treatment, would not necessarily translate to significant changes in its corresponding higher alcohol, isoamyl alcohol, which has a concentration of 88.62 $\mathrm{mg} / \mathrm{L}$, due to their relative abundance (Table 1). Fermentations treated with only sterols resulted in higher levels of acetate esters, with the $\beta$-sitosterol treatments having the highest concentrations (Table 1, Fig. 4A). This is illustrated by isoamyl acetate which showed a $100 \%$ increase, relative to the untreated control, when treated with $\beta$-sitosterol compared to the $70 \%$ increase with ergosterol. It is possible that the abundance of sterols may also contribute to an increased number of lipid particles in which alcohol acetyl transferase is localised (Verstrepen et al., 2004), further contributing to this positive response.

\section{Medium-chain fatty acids (MFA) and their ethyl esters}

Medium-chain fatty acid (hexanoic acid, octanoic acid, and decanoic acid) concentrations at the end of alcoholic fermentation (Fig. 3E, Table 1) also displayed responsiveness to oleic acid supplementation. This resulted in two distinct profiles, namely, elevated levels of medium chain fatty acids in the case of the sterols (Erg, Phy and ErgPhy) and lower levels in response to any dosage of oleic acid (Fig. 4A). For example, hexanoic acid levels were higher, compared to the untreated control, when treated with $\beta$-sitosterol (15\%), ergosterol (33\%) and ergosterol with $\beta$-sitosterol (23\%) (Fig. $3 \mathrm{E})$, whereas, hexanoic acid levels were lower when treated with oleic acid (45\%) and $\beta$-sitosterol with oleic acid (17\%). This response can be explained, as medium chain fatty acid synthesis is repressed by the presence of extracellular unsaturated fatty acids (Saerens et al., 2010). In the absence of exogenous lipids, fatty acid biosynthesis is activated and once oxygen has been depleted, the accumulation of saturated fatty acids results in the release of medium chain fatty acids from the fatty acid synthase complex (Dufour et al., 2003).

Although the ethyl ester concentrations were below the limit of quantification, their production trends mirror that of the medium chain fatty acids (Fig. 3F), as its production is limited by the precursor concentrations, medium chain fatty acid (acyl-CoA) and ethanol, as well as enzyme activity (Saerens et al., 2008). 
(A)

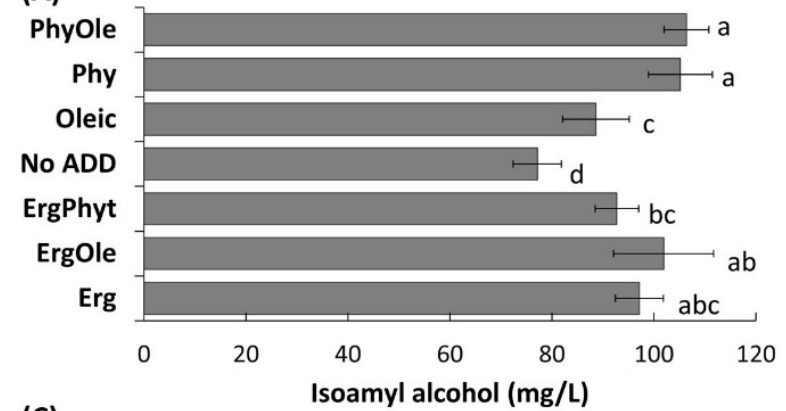

(C)

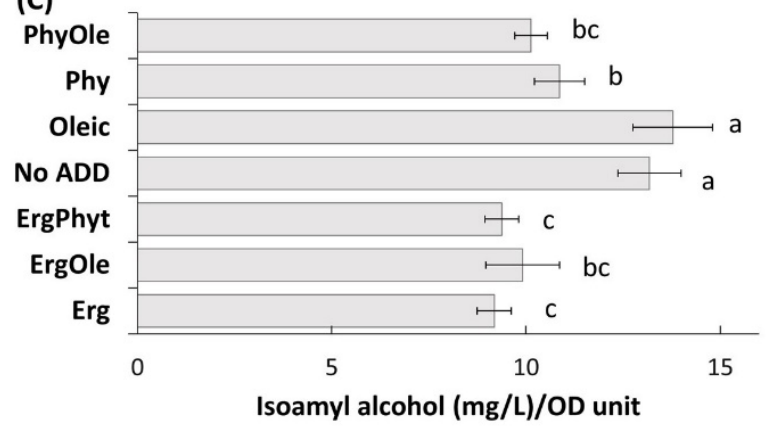

(E)

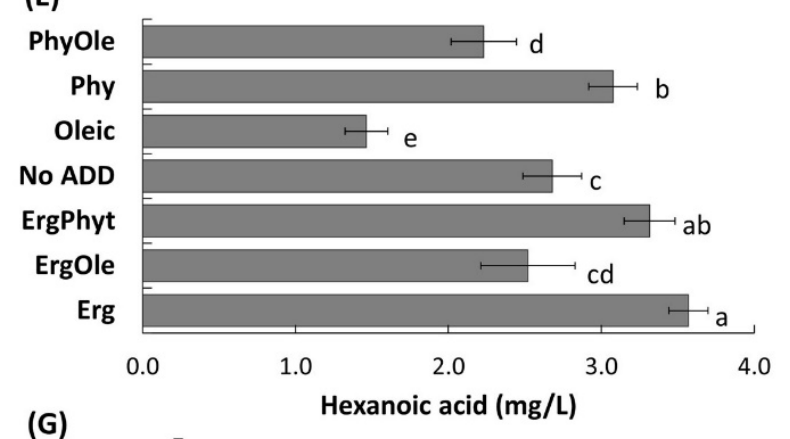

(G)

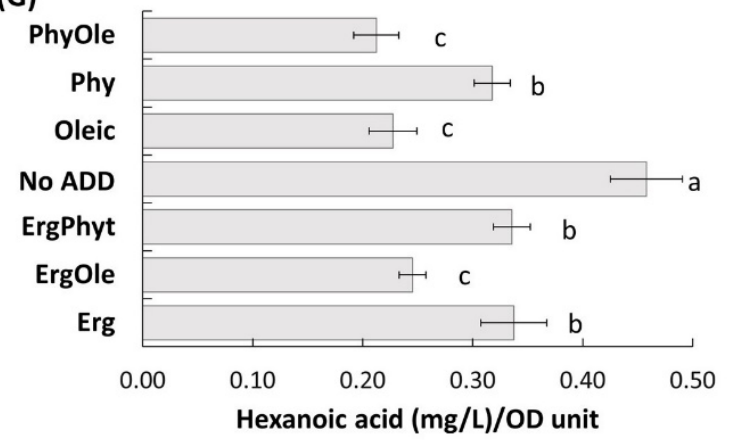

(B)
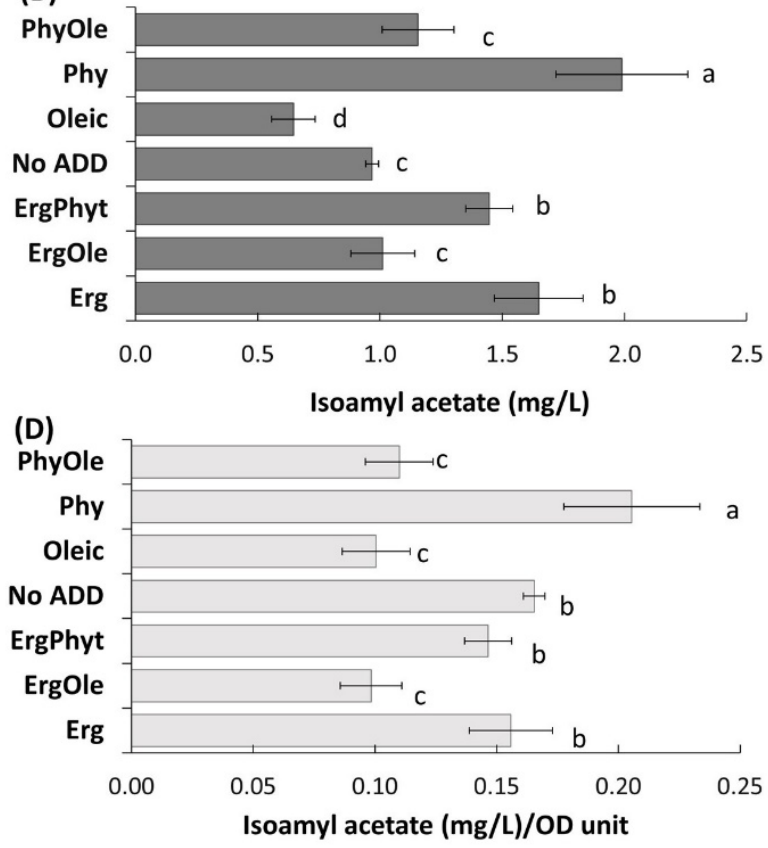

(F)

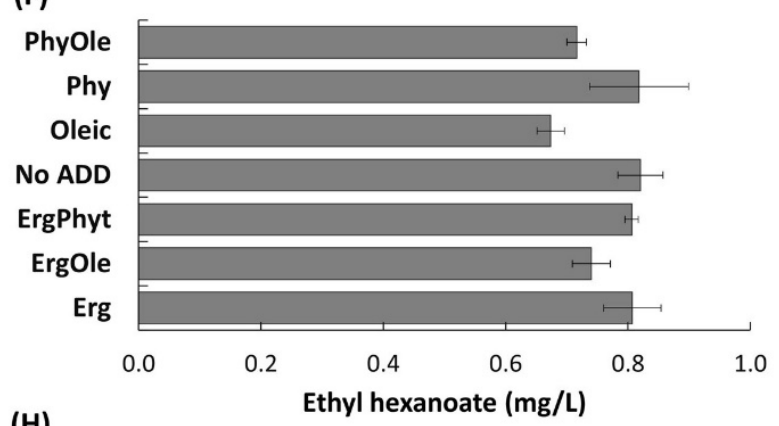

(H)

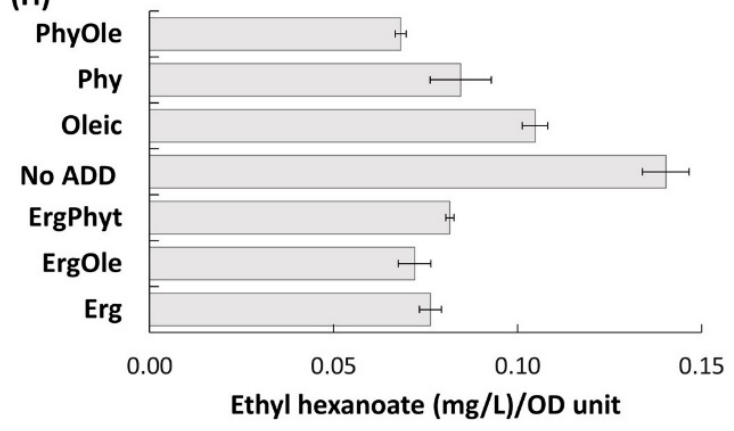

FIGURE 3

The impact of anaerobic factors, as determined by GC-FID (dark grey) and after normalization with OD measurements (light grey), respectively, on a subset of volatile aromas. This includes the production of (A and C) isoamyl alcohol, (B and D) isoamyl acetate, $(\mathrm{E}$ and $\mathrm{G})$ hexanoic acid, and $(\mathrm{F}$ and $\mathrm{H})$ ethyl hexanoate. The anaerobic factors contain ergosterol (Erg), $\beta$-sitosterol (Phy), and oleic acid (Ole) added independently, in addition to their use in combinations with each other. The error bars denote the standard deviation between triplicate treatments, and the letters the significant differences as calculated by Fisher's LSD $(\mathrm{P}<0.05)$.

Following normalization with yeast biomass, the untreated control showed elevated levels of medium chain fatty acids and their ethyl esters (Fig. 3G and H, Fig. 4B), which is possibly indicative of an attempt to enhance the unsaturated fatty acid content of the cell to bolster its stress tolerance before oxygen is depleted. Additionally, the application of oleic acid, regardless of the combination, resulted in similar lower levels of medium chain fatty acids (Fig. 3G).

The most notable difference between the ergosterol and $\beta$-sitosterol treatments is the significant reduction in acetic acid levels observed due to $\beta$-sitosterol (Phy and PhyOle) addition (Fig. 4A), which has been reported previously by other 


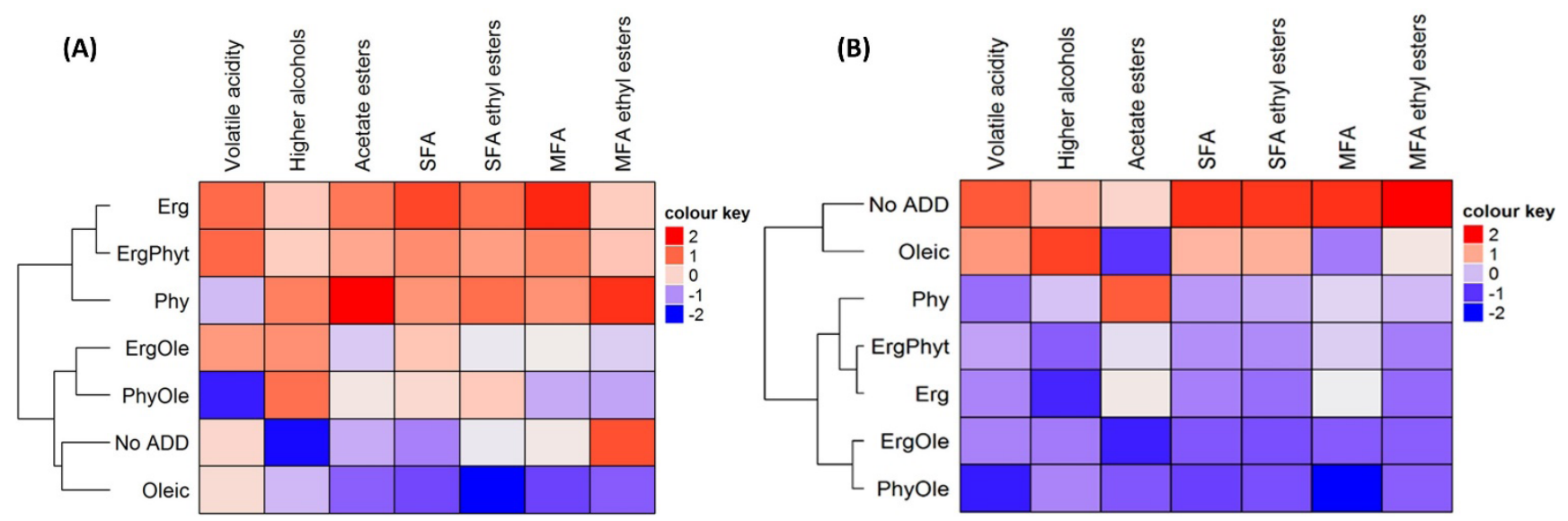

FIGURE4

A comparative overview of the impact of anaerobic factors on the volatile profiles before and after normalization with OD measurements. The impact of anaerobic factors, as determined by GC-FID (A) and after normalization with OD measurements (B), respectively, on volatile acidity (ethyl acetate and acetic acid), $\Sigma$ higher alcohols, $\Sigma$ acetate esters, $\Sigma$ short chain fatty acids (SFA), $\Sigma$ SFA ethyl esters, $\Sigma$ medium chain fatty acids (MFA) and $\Sigma$ MFA ethyl esters levels. The anaerobic factors contained ergosterol (Erg), $\beta$-sitosterol (Phy), and oleic acid (Ole) added independently, in addition to their use in combinations with each other. High values are coloured in red and low blue and the colour intensity represents variation in the levels across the colour scale.

authors (Rollero et al., 2014; Ochando et al., 2017). Here we show that this reduction in volatile acidity is lost when ergosterol is paired with $\beta$-sitosterol. It has already been reported that phytosterol additions also promote the production of succinic acid (Rollero et al., 2014; Ochando et al., 2017), rather than acetic acid, potentially as a means to regenerate NADH. Additionally, when compared to $\beta$-sitosterol, ergosterol resulted in a significant increase in the production of hexanoic acid and decanoic acid (Table 1, Fig. 5A). In part, this may be due to differences in acetyl-CoA metabolism: Phytosterol supplementation has been shown to divert acetyl-CoA to the TCA cycle (succinic acid synthesis) (Rollero et al., 2014; Ochando et al., 2017) and our data suggest that ergosterol may direct acetyl CoA towards fatty acid synthesis. Furthermore, the data trends suggest that the combined use of $\beta$-sitosterol and ergosterol resulted in profiles more similar to ergosterol than $\beta$-sitosterol (Fig. 4A). It is possible that the cell preferentially incorporates ergosterol into the cell membranes and stores the phytosterol (Luparia et al., 2004) for later use. The sterols paired with unsaturated fatty acids were more similar to the untreated control and the oleic acid only treatments (Fig. 4A). This is primarily due to the comparative decrease in acetate ester, medium chain fatty acid and ethyl ester synthesis due to oleic acid supplementation.

\section{CONCLUSIONS}

To our knowledge, this is the first time that the impact of combinations of sterols and unsaturated fatty acids have been compared in this manner. Irrespective of application, oleic acid lowered the production of medium chain fatty acids, as well as ethyl and acetate esters, whereas the specific sterol used altered the production of volatile fatty acids or organic acids. The data illustrates the profound impact that the lipid composition has on fermentation kinetics and aroma production. Moreover, it highlights the importance of managing the lipid composition through the addition of inactivated dried yeast (yeast hulls) or the retention of grape solids, as it may potentially have an unpredictable impact on aroma. This study provides a preliminary data-set highlighting the potential of a more in-depth exploration of the impact of sterol and fatty acid interactions on cell compositional changes. Future work could certainly use chemical filiation experiments to track these compositional changes and their subsequent sensory impact.

\section{LITERATURE CITED}

Aguilera, F., Peinado, R.A, Millán, C., Ortega, J.M. \& Mauricio, J.C., 2006. Relationship between ethanol tolerance, $\mathrm{H}^{+}$-ATPase activity and the lipid composition of the plasma membrane in different wine yeast strains. Int. J. Food Microbiol. 110, 1, 34-42.

Arita, K., Honma, T. \& Suzuki, S., 2017. Comprehensive and comparative lipidome analysis of Vitis vinifera L . cv . Pinot Noir and Japanese indigenous $V$. vinifera L. cv. Koshu grape berries. PLoS ONE. 12, 10.

Bely, M., Sablayrolles, J.M. \& Barre, P., 1990. Description of alcoholic fermentation kinetics: its variability and significance. Am. J. Enol. Vitic. 41, 4, 319-324.

Casalta, E., Cervi, M.F.F., Salmon, J.M.M. \& Sablayrolles, J.M.M., 2013. White wine fermentation: interaction of assimilable nitrogen and grape solids. Aust. J. Grape Wine Res. 19, 1, 47-52.

Casalta, E., Vernhet, A., Sablayrolles, J., Tesnière, C. \& Salmon, J., 2016. Review : Characterization and role of grape solids during alcoholic fermentation under enological conditions. Am. J. Enol. Vitic. 2, 67, 133-138.

Cocito, C. \& Delfini, C., 1997. Experiments for developing selective clarification techniques: sterol and fatty acid loss from grape must related to clarification technique Wine Res. 8, 3, 187-197. 
Daum, G., Lees, N.D., Bard, M. \& Dickson, R., 1998. Biochemistry, cell biology and molecular biology of lipids of Saccharomyces cerevisiae. Yeast $14,16,1471-1510$.

Delfini, C., Cocito, C., Ravaglia, S. \& Conterno, L., 1993. Influence of clarification and suspended grape solid materials on sterol content of free run and pressed grape musts in the presence of growing yeast cells. Am. J. Enol. Vitic. 44, 4, 452-458.

Duan, L., Shi, Y., Jiang, R. \& Yang, Q., 2015. Effects of adding unsaturated fatty acids on fatty acid composition of Saccharomyces cerevisiae and major volatile compounds in wine South African. J. Enol. Vitic. 36, 2, 285-295.

Dufour, J.-P., Verstrepen, K.J. \& Derdelinckx, G., 2003. Brewing yeast. In: T. Boekhout \& V. Robert (eds). Yeasts food. (1st ed.). Woodhead Publishing, Hamburg. pp 347-388.

Fujii, T., Kobayashi, O., Yoshimoto, H., Furukawa, S. \& Tamai, Y., 1997. Effect of aeration and unsaturated fatty acids on expression of the Saccharomyces cerevisiae alcohol acetyltransferase gene. Microbiology 63, 3, 910-915.

Hazelwood, L.A., Daran, J.M., van Maris, A.J.A, Pronk, J.T. \& Dickinson, J.R., 2008. The Ehrlich pathway for fusel alcohol production: a century of research on Saccharomyces cerevisiae metabolism. Appl. Environ. Microbiol. 74, 8, 2259-2266.

Henry, S., 1982. Membrane lipids of yeast: biochemical and genetic study. In: Strathern, J.N., Jones, E.W. \& Broach, J.R. (eds). Molecular Biology of the Yeast Saccharomyces cerevisiae: Metabolism and Gene Expression. Cold Spring Harbor Laboratory Press, New York, pp. 101-158.

Henschke, P.A. \& Jiranek, V., 1993. Yeast: Metabolism of nitrogen compounds. In: G.H. Fleet (ed). Wine Microbiol. Biotechnol. Harwood Academic, Lausanne. pp 77-164.

Lafon-Lafourcade, S., Larue, F. \& Ribereau-Gayon, P., 1979. Evidence for the existence of "survival factors" as an explanation for some percularities of yeast growth, especially in grape must of high sugar concentration. Appl. Environ. Microbiol. 38, 6, 1069-1073.

Louw, L., Roux, K., Tredoux, A., Tomic, O., Naes, T., Nieuwoudt, H.H. \& Van Rensburg, P., 2009. Characterization of selected South African young cultivar wines using FTMIR Spectroscopy, Gas chromatography, and multivariate data analysis. J. Agric. Food Chem. 57, 7, 2623-2632.

Luparia, V., Soubeyrand, V., Berges, T., Julien, A. \& Salmon, J.-M., 2004. Assimilation of grape phytosterols by Saccharomyces cerevisiae and their impact on enological fermentations. Appl. Microbiol. Biotechnol. 65, 1, 25-32.

Ma, M. \& Liu, Z.L., 2010. Mechanisms of ethanol tolerance in Saccharomyces cerevisiae. Appl. Microbiol. Biotechnol. 87, 3, 829-845.

Mannazzu, I., Angelozzi, D., Belviso, S., Budroni, M., Farris, G.A., Goffrini, P., Lodi, T., Marzona, M. \& Bardi, L., 2008. Behaviour of Saccharomyces cerevisiae wine strains during adaptation to unfavourable conditions of fermentation on synthetic medium: Cell lipid composition, membrane integrity, viability and fermentative activity. Int. J. Food Microbiol. 121, $1,84-91$.

Mauricio, J.C., Moreno, J., Zea, L., Ortega, J.M. \& Medina, M., 1997. The effects of grape must fermentation conditions on volatile alcohols and esters formed by Saccharomyces cerevisiae. J. Sci. Food Agric. 75, 2, 155-160.

Mauricio, J.C., Millán, C. \& Ortega, J.M., 1998. Influence of oxygen on the biosynthesis of cellular fatty acids, sterols and phospholipids during alcoholic fermentation by Saccharomyces cerevisiae and Torulaspora delbrueckii. World J. Microbiol. Biotechnol. 14, 3, 405-410.

Ochando, T., Mouret, J.R., Humbert-Goffard, A., Sablayrolles, J.M. \& Farines, V., 2017. Impact of initial lipid content and oxygen supply on alcoholic fermentation in champagne-like musts. Food Res. Int. 98, 87-94.
Piper, P., 1995. The heat shock and ethanol stress responses of yeast exhibit extensive similarity and functional overlap. FEMS Microbiol. Lett. 134, $2-3,121-127$.

Redón, M., Guillamón, J.M., Mas, A. \& Rozès, N., 2011. Effect of growth temperature on yeast lipid composition and alcoholic fermentation at low temperature. Eur. Food Res. Technol. 232, 3, 517-527.

Rollero, S., Bloem, A., Camarasa, C., Sanchez, I., Ortiz-Julien, A., Sablayrolles, J.-M.M., Dequin, S. \& Mouret, J.-R.R., 2014. Combined effects of nutrients and temperature on the production of fermentative aromas by Saccharomyces cerevisiae during wine fermentation. Appl. Microbiol. Biotechnol. 99, 5, 2291-2304.

Rollero, S., Mouret, J.-R., Sanchez, I., Camarasa, C., Ortiz-Julien, A., Sablayrolles, J.-M. \& Dequin, S., 2016. Key role of lipid management in nitrogen and aroma metabolism in an evolved wine yeast strain. Microb. Cell Fact. 15, 1, 32 .

Rosenfeld, E., Beauvoit, B., Blondin, B. \& Salmon, J.-M., 2003. Oxygen consumption by anaerobic Saccharomyces cerevisiae under enological conditions: Effect on fermentation kinetics. Appl. Environ. Microbiol. 69, 1, 113-121.

Sablayrolles, J.M., 2009. Control of alcoholic fermentation in winemaking: Current situation and prospect. Food Res. Int. 42, 4, 418-424.

Saerens, S.M.G., Delvaux, F.R., Verstrepen, K.J., Van Dijck, P., Thevelein, J.M. \& Delvaux, F.R., 2008. Parameters affecting ethyl ester production by Saccharomyces cerevisiae during fermentation. Appl. Environ. Microbiol. $74,2,454-461$.

Saerens, S.M.G., Delvaux, F.R., Verstrepen, K.J. \& Thevelein, J.M., 2010. Production and biological function of volatile esters in Saccharomyces cerevisiae. Microb. Biotechnol. 3, 2, 165-177.

Snoek, I.S. \& Steensma, H., 2007. Factors involved in anaerobic growth of Saccharomyces cerevisiae. Yeast 24, 1, 1-10.

Stukey, J.E., McDonough, V.M. \& Martin, C.E., 1989. Isolation and characterization of OLE1, a gene affecting fatty acid desaturation from Saccharomyces cerevisiae. J. Biol. Chem. 264, 28, 16537-16544.

Taylor, G.T., Thurston, P. a. \& Kirsop, B.H., 1979. The influence of lipids derived from malt spent grains on yeast metabolism and fermentation $\mathrm{J}$. Inst. Brew. 85, 4, 219-227.

Thurston, P.A., Taylor, R. \& Ahvenainen, J., 1981. Effects of linoleic acid supplements on the synthesis by yeast of lipids and acetate esters. J. Inst. Brew. 87, 2, 92-95.

Tumanov, S., Zubenko, Y., Greven, M., Greenwood, D.R., Shmanai, V. \& Villas-Boas, S.G., 2015. Comprehensive lipidome profiling of Sauvignon blanc grape juice. Food Chem. 180, 249-256.

Varela, C., Torrea, D., Schmidt, S.A., Ancin-Azpilicueta, C. \& Henschke, P. A., 2012. Effect of oxygen and lipid supplementation on the volatile composition of chemically defined medium and Chardonnay wine fermented with Saccharomyces cerevisiae. Food Chem. 135, 4, 2863-2871.

Varela, F., Calderon, F., Gonzalez, M.C., Colomo, B. \& Suarez, J.A., 1999. Effect of clarification on the fatty acid composition of grape must and the fermentation kinetics of white wines. Eur. Food Res. Technol. 209, 6, 439444 .

Verstrepen, K.J., Van Laere, S.D.M., Vercammen, J., Derdelinkx, G., Dufour, J.P., Pretorius, I.S., Winderickx, J., Thevelein, J.M. \& Delvaux, F.R., 2004. The Saccharomyces cerevisiae alcohol acetyl transferase Atflp is localized in lipid particles. Yeast 21, 4, 367-377.

Yoshioka, K. \& Hashimoto, N., 1981. Ester formation by alcohol acetyltransferase from brewers' yeast. Yeast 45, 10, 2183-2190. 
You, K.M., Rosenfield, C. \& Knipple, D.C., 2003. Ethanol tolerance in the yeast Saccharomyces cerevisiae is dependent on cellular oleic acid content Appl. Environ. Microbiol. 69, 3, 1499-1503.
Zara, G., Bardi, L., Belviso, S., Farris, G.A., Zara, S. \& Budroni, M., 2008 Correlation between cell lipid content, gene expression and fermentative behaviour of two Saccharomyces cerevisiae wine strains. J. Appl. Microbiol. 104, 3, 906-914. 